\title{
Study of the Dependence of the Plateau Shape for Type II Supernovae on Metallicity
}

\author{
Goldshtein A. A. ${ }^{1 *}$, Blinnikov S. I. ${ }^{2 * * 3,4}$ \\ ${ }^{1}$ St. Petersburg State University, Ul'yanovskaya ul. 1, Petrodvoretz, \\ St. Petersburg, 198504 Russia \\ 2 Alikhanov Institute for Theoretical and Experimental Physics, ul. Bol'shaya \\ Cheremushkinskaya 25, Moscow, 117218 Russia \\ ${ }^{3}$ Sternberg Astronomical Institute, Moscow State University, \\ Universitetskii pr. 13, Moscow, 119992 Russia \\ ${ }^{4}$ Center for Fundamental and Applied Research, Dukhov All-Russia Research Institute of \\ Automatics, ul. Sushchevskaya 22, Moscow, 127055 Russia
}

\begin{abstract}
We consider the effect of a change in the rate of flux decline in the $U$ band for type II-P supernovae (SNe IIP) as a function of metallicity $Z$. Based on this effect, we propose a new method to determine the photometric redshift from the SN IIP light curve in the $U$ band. Using the STELLA code, we have constructed model light curves in different bands for different redshifts $z=0.0,0.1$, and 0.3 as the metallicity in the models decreases from $Z \sim 10^{-3}$ to $\sim 10^{-6}$. The flux in the $U$ band is shown to reach a plateau at the lowest metallicities. We consider the influence of other parameters as well: the presupernova mass and the mass of radioactive nickel-56.
\end{abstract}

* E-mail: <angold107@gmail.com>

** E-mail: <Sergei.Blinnikov@itep.ru> 


\section{INTRODUCTION}

Observations of classical type II plateau supernovae, SNe IIP, show that in the $U, R$, $V, I$, and $Z$ bands the fluxes do remain constant for two or three months, i.e., form a "plateau" that gave the name to this type. At the same time, the fluxes in the $U$ band decline steeply linearly (see, e.g., Leonard et al. 2002; Hicken et al. 2017). A less steep decline is observed in the $B$ band.

This behavior of the light curves in different bands cannot be described using the "gray" approximation in computations, which is used, for example, in the open source SNEC code (Morozova et al. 2015), where the assumption about blackbody radiation by the photosphere (with bolometric corrections) is applied to construct the light curves in different bands. The light curve constructed in this way for the $U$ band does not reproduce the observations, because the enhanced absorption by metals in the ultraviolet in the cold above-photosphere layers of the SN ejecta is required to be taken into account here.

One of the first successful computations of the effect of a linear flux decline in the $U$ band was the application of the EDDINGTON code for SNe IIP (Eastman et al. 1994). The STELLA code (Blinnikov and Sorokina 2000; Blinnikov et al. 2006), which does not use the gray approximation, but is based on multigroup radiative transfer, allows the observed effect to be described realistically.

Depending on the presupernova parameters, the slope of the linear decline in the $U$ band can change. Baklanov et al. (2005) noticed how the metallicity of the presupernova envelope affects the light-curve shape or, more specifically, the lower the metallicity, the slower the decline. In this paper we consider in detail the influence of this parameter with the goal of a practical application of the gained knowledge to supernovae with a low metallicity in their envelopes. On the other hand, the redshift $z$ also affects the slope of the light curve in the observed bands, because at a sufficiently large $z$, for example, the observed $V$ band will correspond to the $U$ band in the supernova rest frame and there will be a linear decline instead of the plateau in the $V$ band. Consequently, the metallicity and the redshift can be related between each other: if the metallicity is known from other observations, then $z$ can be estimated. Conversely, if the redshift of the galaxy inwhich the supernova exploded is known, then the metallicity of its envelope can be estimated.

The redshifts of galaxies and supernovae are much more difficult to measure than their 
photometric fluxes. Therefore, methods for measuring the socalled photometric redshifts have been proposed long ago for galaxies (Koo 1985; Padmanabhan et al. 2005). Attempts to determine the redshift from supernovae are known, but they concern only type Ia. For example, one can use the $g^{\prime}, r^{\prime}, i^{\prime}$, and $z^{\prime}$ bands and determine the redshift from the fluxes at maximum light in these bands via the fitted coefficients (Wang 2007; Wang et al. 2015). Using the SALT2 code, Palanque-Delabrouille et al. (2018) constructed light curves for SNe Ia in the same bands. The boundaries for the redshift were determined for different sets of supernova parameters. Light curves with a given $z$ step were constructed from approximate parameters of the light curve under study and it was compared with them by the $\chi^{2}$ method.

In addition, one can use the knowledge that the cosmological redshift affects the entire spectrum, while absorption affects mostly the blue part of the spectrum and compare the color indices, as was done by Kessler et al. (2010).

Type II supernovae are used for cosmology not as actively as SNe Ia, because their absolute luminosity is lower than that for SNe Ia. However, since this is the largest class of supernovae and since the power of telescopes grows, an independent determination of the redshifts to these objects becomes an increasingly topical problem. In this paper we show that the effect of a change in the slope of the light curve undoubtedly takes place. A more quantitative analysis and lightcurve calibrations for cosmological applications will be performed in our succeeding publications.

\section{MODEL CONSTRUCTION}

In the STELLA code the presupernova model is constructed for a set of specified parameters. The hydrostatic equilibrium equation with the assumption about a weak dependence of the temperature on density, $T \propto \rho^{\alpha}$, is used in the code. For a fully ionized gas and homogeneous chemical composition this hydrostatic state is close to a polytrope with an index $1 / \alpha \approx 0.3$, which satisfactorily describes the evolutionary models, for example, from Tolstov et al. (2016). Such an approach is used in many papers (see, e.g., Utrobin 2007). The deviation from the polytropic model increases in the outer layers due to recombination and inhomogeneous chemical composition. A point-like heavy core $0.1 R_{\odot}$ in size is confined at the center of the model. All of the elements, except for ${ }^{56} \mathrm{Ni}$, behind the shock front are assumed to bemixed uniformly. Since the distribution and amount of 
${ }^{56} \mathrm{Ni}$ produced during the explosion affect significantly the luminosity, its distribution is specified as decreasing exponentially toward the outer layers.

Using the STELLA code, we constructed several models, they are listed in Table 1. In the first family of models 1-4 we took standard parameters of a supernova with different metallicities (Fig. 1). Note that the decline becomes less sharp as the metallicity decreases.

Then, we considered another set of parameters with the suffix "nc" in the name (Fig. 2). Apart from an increase in the total mass and virtual zeroing of the nickel-56 mass, the relative mass of the core with heavy elements was artificially reduced by a factor of 10. Thus, we can investigate the influence of metallicity in the envelope on the light curve in pure form. Compared to the first family, the dependence on $Z$ in this set of models is much stronger and at $Z=4 \times 10^{-6}$ the plateau in the $U$ band has a very small slope. In the next models ("nc3") we used the same parameters, but the number of zones was increased for a higher accuracy. In particular, the oscillations in the region 20-50 days for model 13 were slightly smoothed (Fig. 3). In the models with the suffix "c3t" (see Fig. 10 below) the boundary of the approximations of the inner and outer layers was shifted outward. Thismade the light curves smoother.

In different photometric systems for the $U$ band (Fig. 6) the light curves look similar and the dependence of the slope on metallicity is confirmed. In the $R, I$, and $V$ bands the slope is virtually constant at different metallicities $Z$ and this effect is imperceptible; therefore, they are not worth considering. For example, models 1 and 4 (Fig. 9) exhibit a change in the slope at different metallicities in the $U$ band, but in the $V$ band it remains approximately the same. The same effect is also retained for models 14 and 17 (Fig. 10). This effect can be observed in the $B$ band, but it manifests itself not as clearly as in $U$.

In the $R, I$, and $V$ bands this effect does not manifest itself at different metallicities $Z$, i.e., the plateau shape is retained (see, e.g., Fig. 5). It follows from Fig. 10 that a change in $Z$ affects very weakly the plateau shape in the $V$ band. In the $B$ band the effect is not as strong as in $U$.

It follows from the graphs that under the same conditions a decrease in metallicity leads to an increasingly slow decline in the $U$ band and, hence, we can try to find an application of this dependence. Figure 5 shows the light curves constructed in different bands for models 14 and 17 with different redshifts: $z=0,0.1,0.3$. As $z$ increases, the $U$ 
and $B$ light curves have a faster decline due to the redshift. Note that at $Z=10^{-6}$ and $z=0.3$ the shape of the light curves is similar to that of the model with $Z=10^{-3}$ and $z=0$. Therefore, given the slope, we cannot unambiguously determine the metallicity. However, this becomes possible at a known redshift. Conversely, the redshift $z$ can be found from the slope and metallicity. This opens a simple way for estimating the metallicity or redshift of metal-poor supernovae.

Tolstov et al. (2016) considered the light-curve shape at low or zero metallicity, where the model with zerometallicity, amass of $25 M_{\odot}$, and $M_{\mathrm{Ni}}=0,10^{-3}$, and $10^{-1} M_{\odot}$ is of interest to us. In this model at the first and second values of the nickel-56 mass the plateau is kept at the same level, as in our M25_Z4e- 3nc3 and M25_Z4e-6nc3 models. The possibility to determine the parameters from the plateau length is discussed in the same paper, but it is often unlikely to catch a supernova explosion before the plateau. In our paper we consider precisely the slope and, therefore, the time of the first measurement plays no major role.

We know yet another paper (Dessart et al. 2013), where different metallicities were considered for SNe IIP, but in a narrower range than that in this paper $(Z=$ 0.04, 0.02, 0.008, 0.002). In their models the slope in the $U$ band is approximately the same and only slightly smaller in the model with $Z=0.002$. At such a negligible difference it is impossible to establish a clear dependence. Therefore, this method cannot be applied to models with medium or high metallicities. In addition, Dessart et al. (2013) considered models with different kinetic energies $\left(E_{\text {kin }}=0.6,1.3,2.9\right)$ at solar metallicity and with identical other parameters. The slope also changes in them and, therefore, in future it is worth checking the influence of energy at low metallicities as well.

Recently, Potashov and Yudin (2020) have investigated the influence of metallicity on the important effect of time-dependence during the formation of spectral lines in SNe IIP discovered by Utrobin and Chugai (2002, 2005).

As is well known, for example, from Imshennik and Nadyozhin (1988) and Utrobin (2007), the lightcurve segment of interest to us follows the passage of various supernova explosion stages (see Fig. 6 in Utrobin (2007)): after $t_{1}$ at shock breakout adiabatic expansion begins (from $t_{1}$ to the point $t_{2}$ at which the decline rate slows down and the plateau begins). It is here at the cooling wave and recombination phase from $t_{2}$ to the end of the plateau $t_{3}$ that the light-curve segment being studied in this paper is located. 
It follows from Figs. 7 and 8 that the velocity at the photospheric level is quite typical for SNe IIP and a low metallicity does not affect it.

The physical causes of the dependence of the plateau decline rate on metallicity are fairly complex and require a careful consideration. This is planned to be done in our subsequent paper.

Table 1: Basic parameters

\begin{tabular}{|c|c|c|c|c|c|}
\hline No. & Model & $\mathrm{Z}$ & $M, M_{\odot}$ & $M_{\mathrm{Ni}}, M_{\odot}$ & Number of zones \\
\hline 1 & M15_Ni004 & $4 \times 10^{-3}$ & 15 & 0.04 & 100 \\
\hline 2 & M15_Ni004Z4e-4 & $4 \times 10^{-4}$ & 15 & 0.04 & 100 \\
\hline 3 & M15_Ni004Z4e-5 & $4 \times 10^{-5}$ & 15 & 0.04 & 100 \\
\hline 4 & M15_Ni004Z4e-6 & $4 \times 10^{-6}$ & 15 & 0.04 & 100 \\
\hline 5 & M15_Ni1e-8Z4e-6 & $4 \times 10^{-6}$ & 15 & $10^{-8}$ & 100 \\
\hline 6 & M25_Z4e-3nc & $4 \times 10^{-3}$ & 25 & $10^{-8}$ & 100 \\
\hline 7 & M25_Z4e-4nc & $4 \times 10^{-4}$ & 25 & $10^{-8}$ & 100 \\
\hline 8 & M25_Z4e-5nc & $4 \times 10^{-5}$ & 25 & $10^{-8}$ & 100 \\
\hline 9 & M25_Z4e-6nc & $4 \times 10^{-6}$ & 25 & $10^{-8}$ & 100 \\
\hline 10 & M25_Z4e-3nc3 & $4 \times 10^{-3}$ & 25 & $10^{-8}$ & 300 \\
\hline 11 & M25_Z4e-4nc3 & $4 \times 10^{-4}$ & 25 & $10^{-8}$ & 300 \\
\hline 12 & M25_Z4e-5nc3 & $4 \times 10^{-5}$ & 25 & $10^{-8}$ & 300 \\
\hline 13 & M25_Z4e-6nc3 & $4 \times 10^{-6}$ & 25 & $10^{-8}$ & 300 \\
\hline 14 & M25_Z4e-3nc3t & $4 \times 10^{-3}$ & 25 & $10^{-8}$ & 300 \\
\hline 15 & M25_Z4e-4nc3t & $4 \times 10^{-4}$ & 25 & $10^{-8}$ & 300 \\
\hline 16 & M25_Z4e-5nc3t & $4 \times 10^{-5}$ & 25 & $10^{-8}$ & 300 \\
\hline 17 & M25_Z4e-6nc3t & $4 \times 10^{-6}$ & 25 & $10^{-8}$ & 300 \\
\hline
\end{tabular}




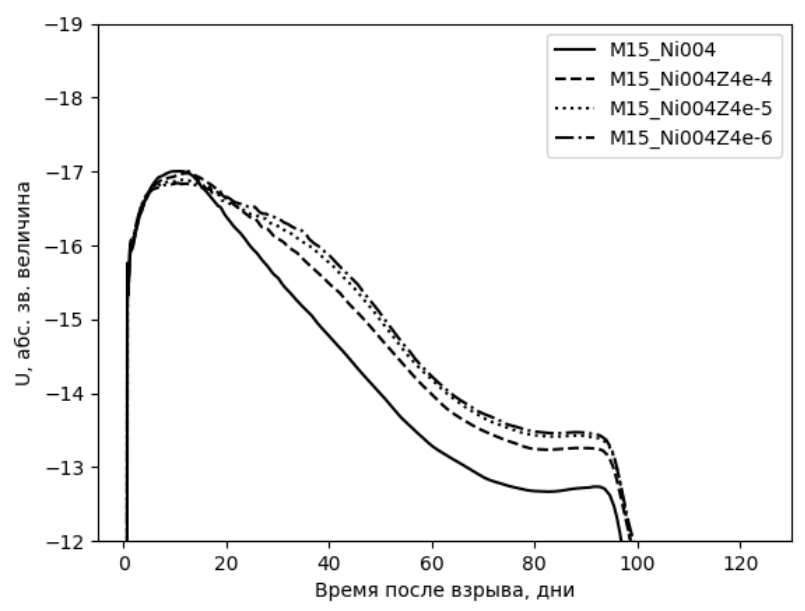

Figure 1: Light curves for models $1-4$ in the $U$ band. X axis: Time after explosion, days. $\mathrm{Y}$ axis: $\mathrm{U}$, abs. magnitude

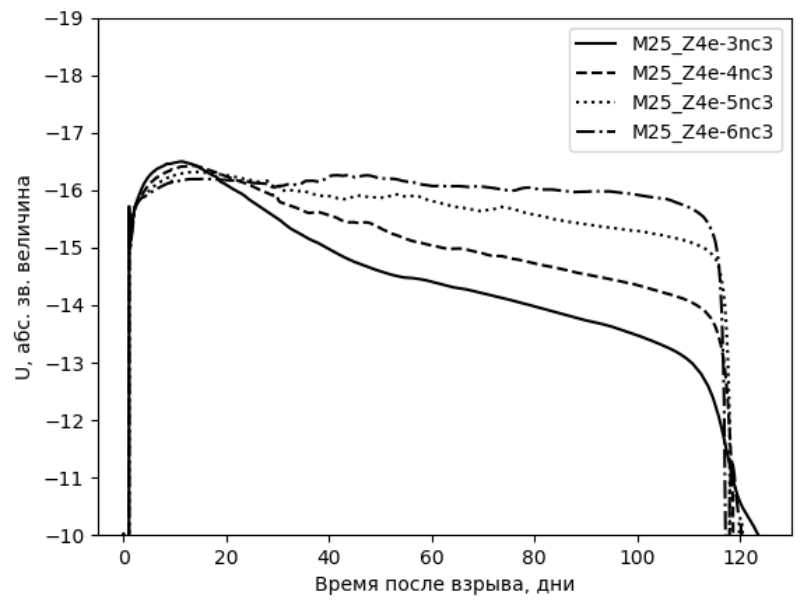

Figure 3: Light curves for models 10-13 in Figure 4: Light curves for models 14-17 in the $U$ band.

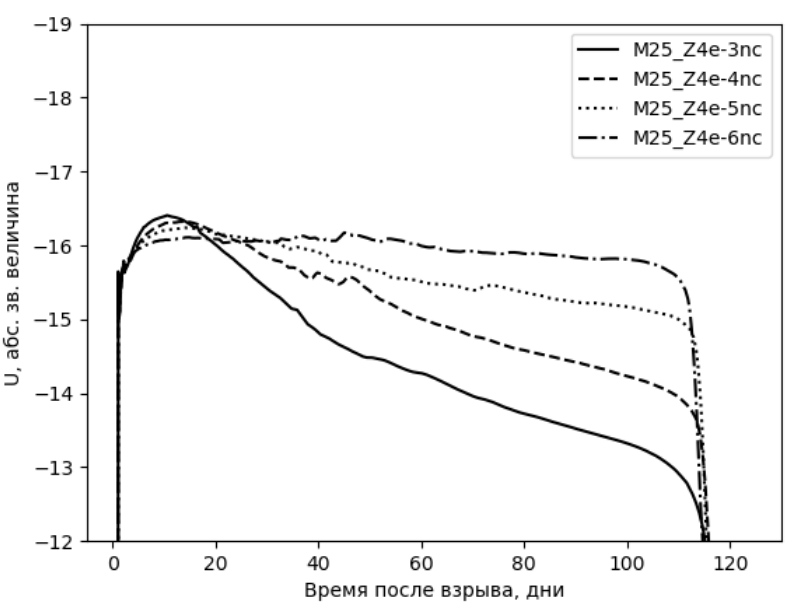

Figure 2: Light curves for models $6-9$ in the $U$ band.

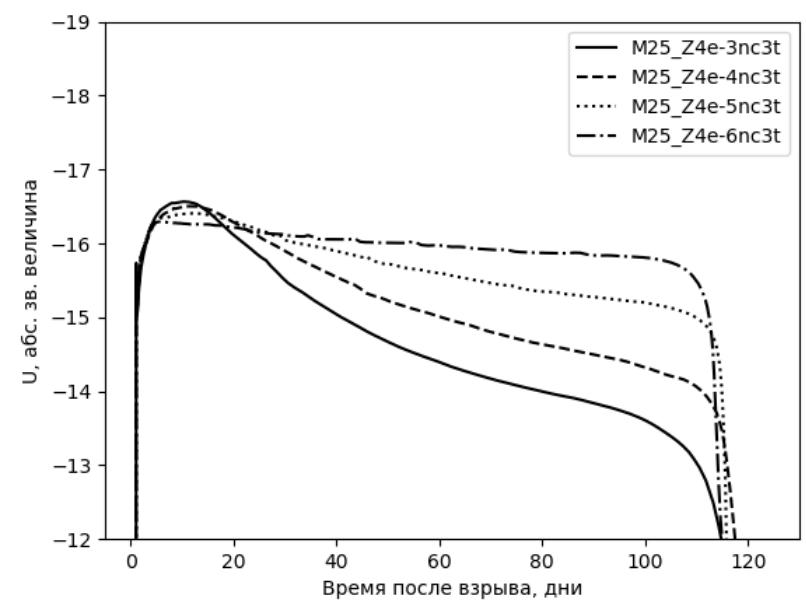

\section{CONCLUSIONS}

In this paper we considered the change in the shape of the $U$-band light curve as a function of low metallicity, with all other parameters being fixed. The light curve was shown to change similarly in this case. Therefore, by analyzing the slope after the peak, one can find the redshift given the metallicity or the metallicity given the redshift. This method does not depend on the start date of observations; hence it can be very convenient 
in view of the difficulty of detecting metal-poor supernovae due to their low luminosity (Tolstov et al. 2016). In addition, this rule holds in different photometric systems for bands close to $U$.

A minor change of the slope in the $U$ band occurs for stars of nearly solar metallicity. In this case, the photometric redshift can be estimated by comparing the slopes in the $U$, $B, V, R$, and $I$ bands. First of all, based on long-wavelength bands, one should make sure that the supernova does not belong to the SN IIL class. Such supernovae observed locally, i.e., at low redshifts, exhibit a linear decline in the $V$ band. How can they be distinguished from distant SNe IIP, i.e., from the flux decline stemming from the fact that at a high redshift $z$ the rest-frame fluxes from the $B$ and $U$ bands fall into the observed $\mathrm{V}$ band? For this purpose, the fluxes in the $R, I$ bands and the near infrared should be examined. In SNe IIP they will reach a horizontal plateau, while in well-observed SNe IIL they have the same linear decline as that in $V$ (Faran et al. 2014; Bose et al. 2018). If the long-wavelength fluxes reach a plateau, then the decline in the $V$ band will be due to the redshift and $z$ can be measured after an appropriate calibration. Since such telescopes as LSST will discover approximately as many SNe IIP as SNe Ia (LSST Science Collaboration 2009), it will be possible to compare the derived photometric redshifts of SNe IIP with the redshifts of their host galaxies en masse. Furthermore, it should also be kept in mind that other parameters, such as the radius, the mass, and the explosion energy, can also affect the light-curve shape. Therefore, this method should be applied when the main characteristics have been determined for sure.

Despite the fact that the values of $Z$ under consideration are very low, stars with such a metallicity do exist in the nearest neighborhoods. For example, Nordlander et al. (2019) has recently discovered a star with $Z \approx 10^{-8}([\mathrm{Fe} / \mathrm{H}]=-6.2)$ in our Galaxy and, therefore, a consideration of models with such low metallicities is not groundless. Of course, in our neighborhoods there are no massive stars with such low $Z$ that could explode as core-collapse supernovae, but, at the same time, there are very many such massive stars in the first generation and their explosions in the nearest future will be observed with the ground-based and space telescopes under construction. 


\section{ACKNOWLEDGMENTS}

A.A. Goldshtein thanks P.V. Baklanov and M.Sh. Potashov for their help in preparing this paper and M.V. Kostina for the useful discussions. S.I. Blinnikov thanks the Russian Science Foundation for its support of the work on the development of the STELLA code (project no. 19-12-00229). 


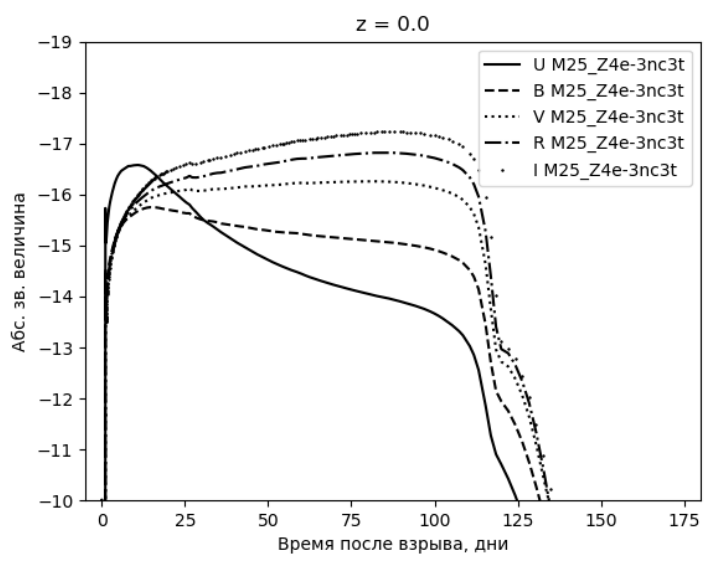

a)

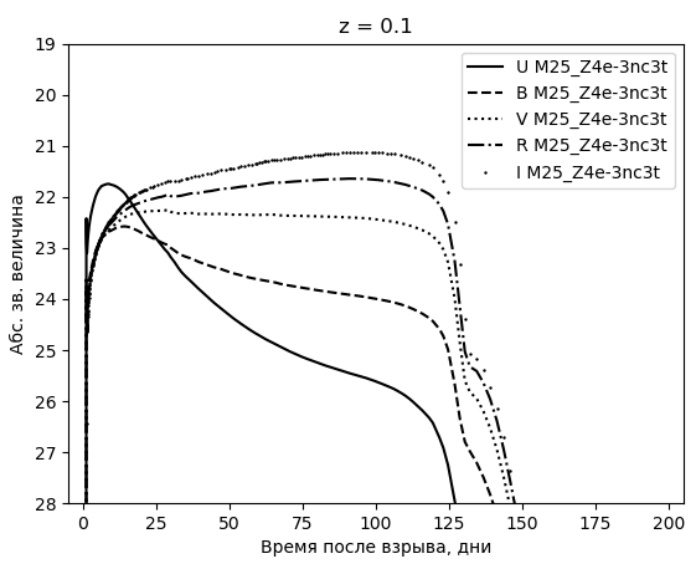

c)

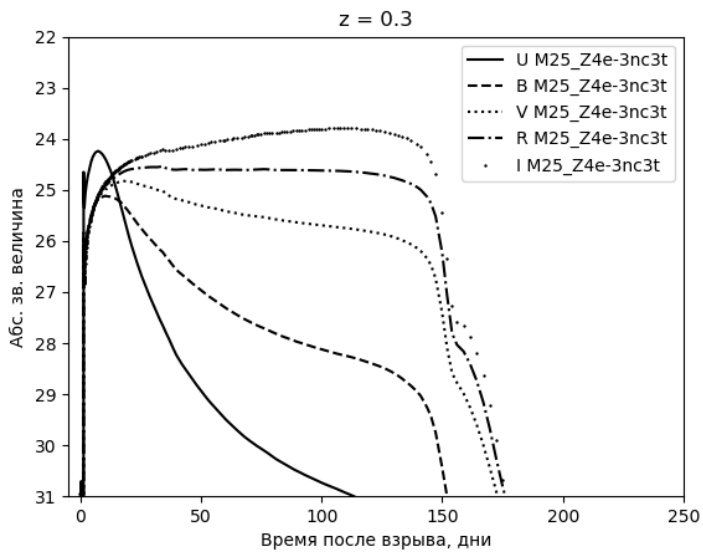

e)

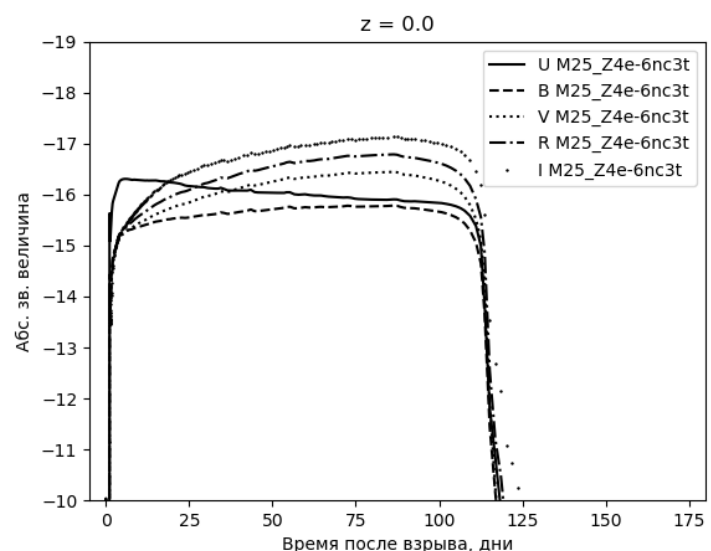

b)

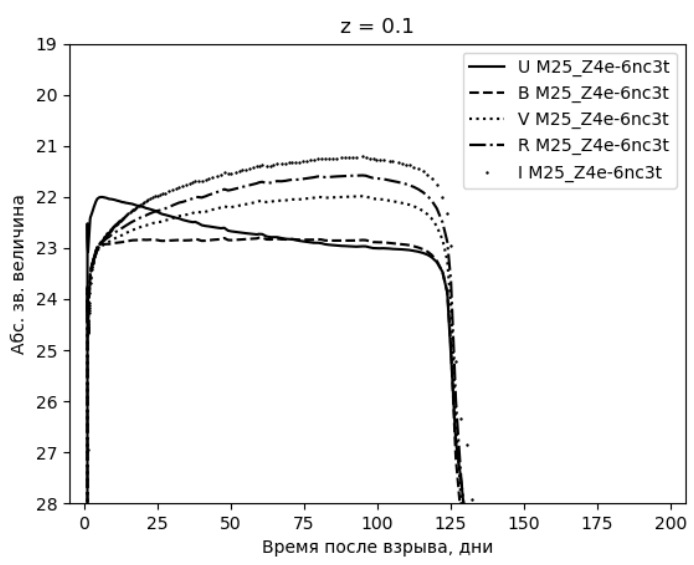

d)

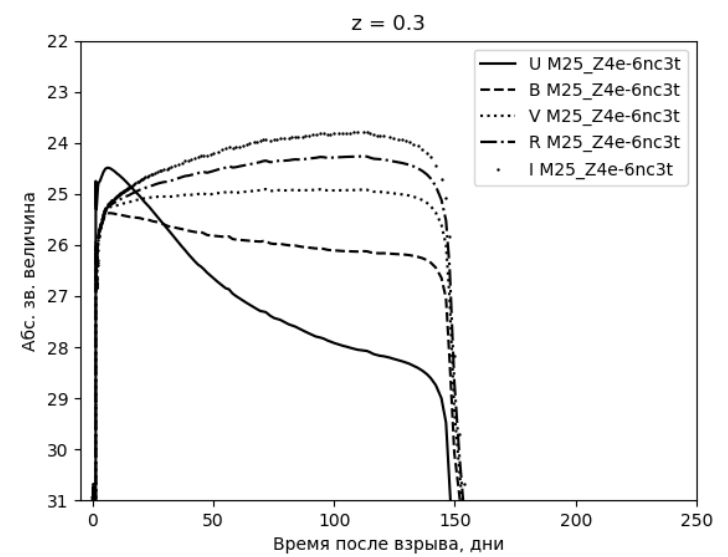

f)

Figure 5: Light curves for extreme metallicities and different redshifts: a) M25_Z4e-3nc3t with $\mathrm{z}=0$, b) M25_Z4e-6nc3t with $\mathrm{z}=0$, c) M25_Z4e-3nc3t with $\mathrm{z}=0.1$, d) M25_Z4e-6nc3t with $z=0.1$, e) M25_Z4e-3nc3t with $z=0.3$, f) M25_Z4e-6nc3t with $z=0.3$. X axis: Time after explosion, days. Y axis: Abs. magnitude 

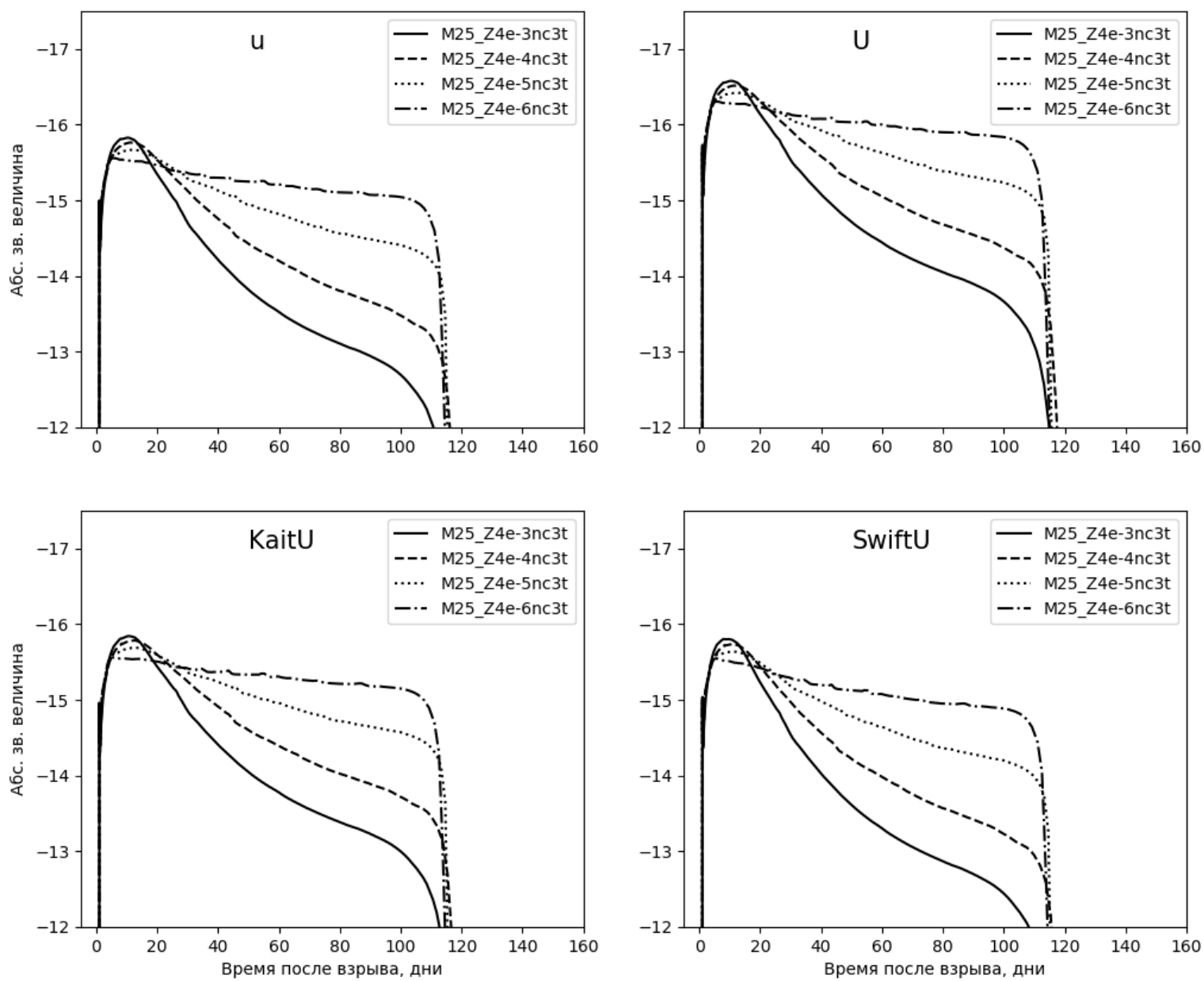

Figure 6: Light curves for models 6-9 in different photometric systems for the $U$ band. $\mathrm{X}$ axis: Time after explosion, days. Y axis: Abs. magnitude 

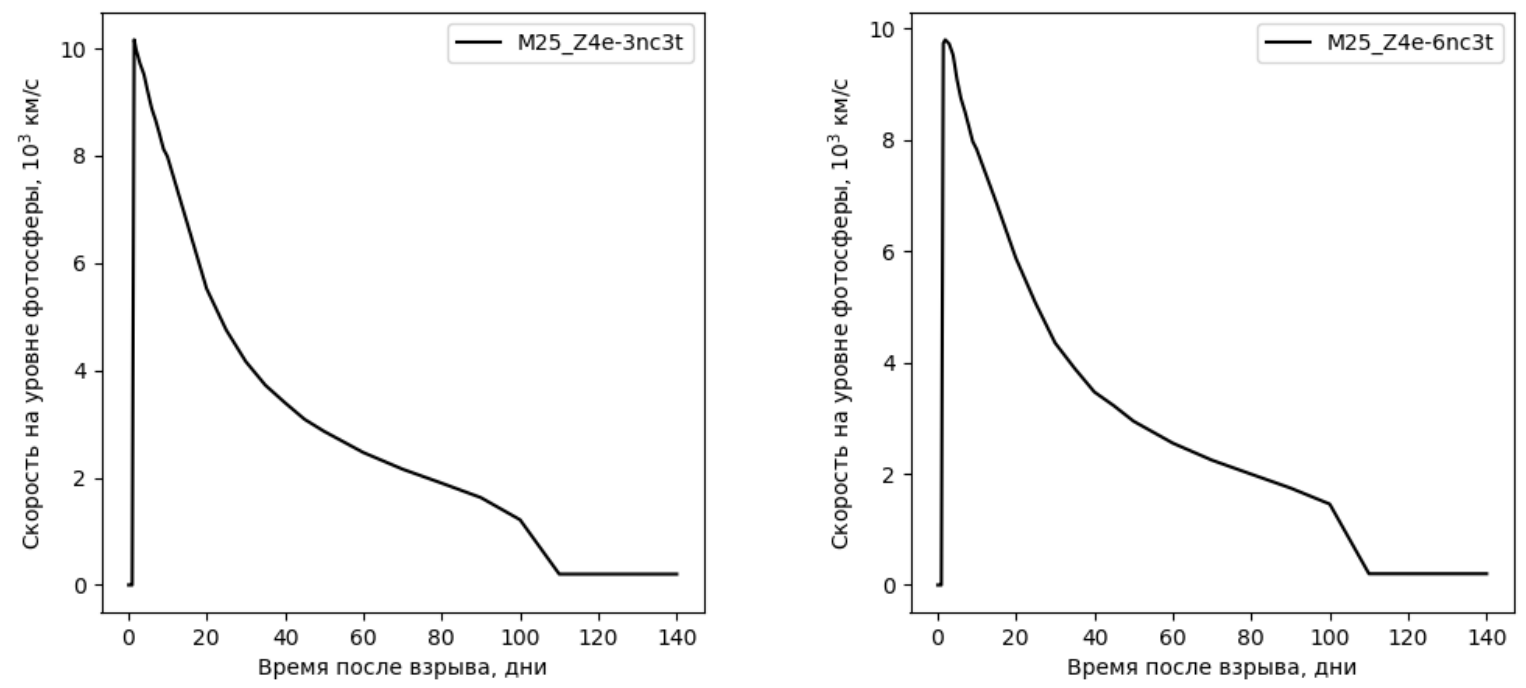

Figure 7: Velocity at the photospheric level Figure 8: Velocity at the photospheric level for model 14 (with a metallicity of $10^{-3}$ ). X for model 17 (with a metallicity of $10^{-6}$ ). X axis: Time after explosion, days. Y axis: axis: Time after explosion, days. Y axis: Velocity at photospheric level, $10^{3} \mathrm{~km} / \mathrm{s} \quad$ Velocity at photospheric level, $10^{3} \mathrm{~km} / \mathrm{s}$
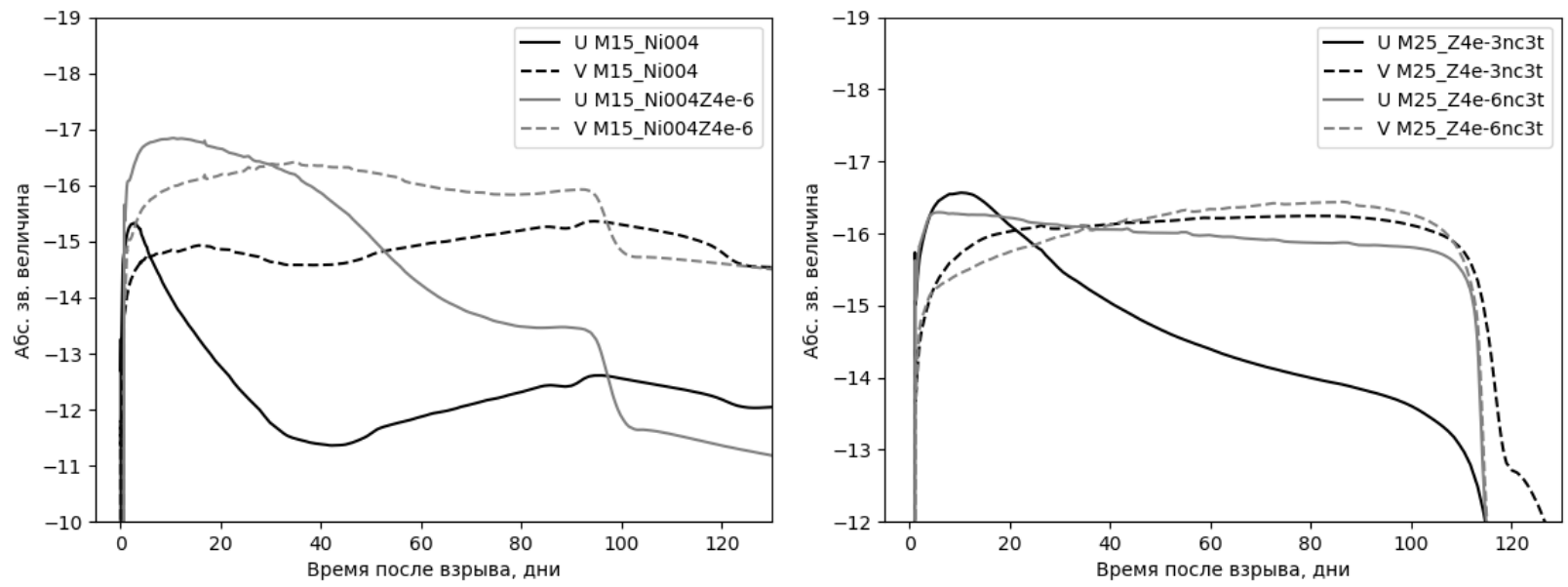

Figure 9: Light curves for models 1 and 4 Figure 10: Light curves for models 14 and 17 in the $U$ and $V$ bands. $\mathrm{X}$ axis: Time after in the $U$ and $V$ bands. $\mathrm{X}$ axis: Time after explosion, days. Y axis: Abs. magnitude explosion, days. Y axis: Abs. magnitude 


\section{REFERENCES}

1. P. V. Baklanov, S. I. Blinnikov, and N. N. Pavlyuk, Astron. Lett., 31, 483 (2005).

2. S. I. Blinnikov, F. K. Ropke, E. I. Sorokina, M. Gieseler, M. Reinecke, C. Travaglio, W. Hillebrandt, and M. Stritzinge, Astronomy and Astrophysics, 453, 229 (2006)

3. S. I. Blinnikov and E. I. Sorokina, Astronomy and Astrophysics, 356, L30 (2000)

4. S. Bose, S. Dong, C. S. Kochanek, A. Pastorello, B. Katz, D. Bersier, J. E. Andrews, J. L. Prieto, et al., The Astrophysical Journal, 862107 (2018)

5. Y.Wang, The Astrophysical Journal Letters, 654, L123 (2007)

6. Y. Wang, E. Gjergo, and S. Kuhlmann, (Y. Wang, E. Gjergo, S. Kuhlmann) MNRAS, 451, 1955 (2015)

7. L. Dessart D. J. Hillier, R. Waldman, and E. Livne, MNRAS, 433, 1745 (2013)

8. V. S. Imshennik and D. K. Nadyozhin, Sov. Sci. Rev., 156, 561 (1988)

9. R. G. Eastman S. E. Woosley, T. A. Weaver, and P. A. Pinto, Pub. Astron. Soc. Pacific, 430, 300 (1994)

10. R. Kessler et al., The Astrophysical Journal, 717, 40 (2010)

11. LSST Science Collaboration 247 colleagues. LSST Science Book, Version 2.0. arXiv e-prints arXiv:0912.0201 (2009)

12. D. C. Koo, Astronomical Journal, 90, 418 (1985)

13. D. C. Leonard, A. V. Filippenko, W. Li, et al., Pub. Astron. Soc. Pacific, 114, 35 (2002)

14. V.Morozova et al., The Astrophysical Journal, 814, 83 (2015)

15. T. Nordlander, M. S. Bessell, G. S. Da Costa, et al., MNRAS, 488, L109 (2019)

16. N. Padmanabhan, T. Bu-davari, D. J. Schlegel, et al., MNRAS, 359, 237 (2005)

17. N. Palanque-Delabrouille, V. Ruhlmann-Kleider, S. Pascal, J. Rich, J. Guy, G. Bazin, P. Astier, C. Balland, et al., Astronomy and Astrophysics, 514, A63 (2010)

18. M. Potashov and A. Yudin, MNRAS, 491, 2674 (2020)

19. A. Tolstov, K. Nomoto, N. Tominaga, M. N. Ishigaki, S. Blinnikov, and T. Suzuki, The Astrophysical Journal, 821, 124 (2016) 
20. V. P. Utrobin and N. N. Chugai, Astron. Lett, 28, 440-447 (2002)

21. V. P. Utrobin, Astronomy and Astrophysics, 461, 233 (2007)

22. V. P. Utrobin and N. N. Chugai, Astronomy and Astrophysics, 441, 271-281 (2005).

23. T. Faran, D. Poznanski, A. V. Filippenko, et al., MNRAS, 445, 554-569 (2014)

24. M. Hicken, A. S. Friedman, S. Blondin, P. Challis, P. Berlind, M. Calkins, G. Esquerdo, Th. Matheson, et al., The Astrophysical Journal Supplement, 233, 6 (2017) 